\title{
Colon cancer and immunotherapy-can we go beyond microsatellite instability?
}

\author{
Rimini Breakstone \\ Lifespan Cancer Institute, Warren Alpert School of Medicine at Brown University, Providence, RI, USA \\ Correspondence to: Rimini Breakstone. Lifespan Cancer Institute, Warren Alpert School of Medicine at Brown University, 164 Summit Avenue, \\ Providence, RI 02906, USA. Email: Rimini.breakstone@lifespan.org.
}

\begin{abstract}
Immune checkpoint blockade (ICB) has changed the landscape of cancer therapy in multiple tumor types since the first agent, Ipilimumab, was first FDA approved for the treatment of metastatic melanoma in 2011. Its role in GI Cancers, particularly in colon cancers, has not been as robust as in other tumor types but select patients with DNA mismatch repair defects, even those who has progressed on multiple standard chemotherapeutic regimens have demonstrated significant, almost unprecedented, responses in this multidrug refractory population. Unfortunately, these cases represent only a small percentage of colon cancer patients with little efficacy in the $95 \%$ of metastatic colon cancers who have proficient DNA mismatch repair. Multiple strategies have been, and are currently being, evaluated to determine the potential benefits of this drug class to microsatellite stable (MSS) patients.
\end{abstract}

Keywords: Colorectal cancer (CRC); immune check point blockade; PD-1; PD-L1; CTLA-4; microsatellite stable (MSS); proficient DNA mismatch repair

Received: 01 August 2019; Accepted: 11 December 2019; Published: 05 January 2021.

doi: $10.21037 /$ tgh.2020.03.08

View this article at: http://dx.doi.org/10.21037/tgh.2020.03.08

\section{Introduction}

Colorectal cancer (CRC) is second most common cause of cancer deaths in men and women in the United States with an estimated 51,020 deaths projected for 2019. There will be an estimated 145,600 new cases of CRC in 2019 and approximately $22 \%$ of new cases are metastatic. Though death rates have improved by an average of $2.2 \%$ each year over 2007-2016, overall prognosis remains poor with 5-year survival in Stage IV colon cancer only approximately 14\% (1). Since the development of 5-Fluorouracil in the late 1950s by Heidelberger and colleagues (2), the backbone of metastatic CRC (mCRC) treatment has been systemic chemotherapy. In the $21^{\text {st }}$ century, targeted treatments began to add to this treatment paradigm. Monoclonal antibodies targeting vascular endothelial growth factor receptors (VEGFR) and epidermal growth factors receptors (EGFR), either alone, or in combination with chemotherapy, are now part of the standard of care in mCRC. The newest class of therapy that has added substantially to management of mCRC is immune checkpoint inhibitors (ICIs). To date, the immune checkpoint pathways of highest clinical significance have been programmed cell death 1 (PD-1) and cytotoxic T-lymphocyte associated antigen 4 (CTLA-4). Ipilimumab, a CTLA-4 inhibitor, was the first FDA approved ICI in 2011 for treatment of metastatic melanoma (3). Since then, between October 2015 and March 2019, the FDA has approved ICIs in 14 different cancer types. The first agent the FDA approved for treatment of colorectal cancer, specifically microsatellite unstable - high (MSI-H) or deficient mismatch repair (dMMR) colorectal cancer that had progressed following treatment with fluoropyrimidine regimen, was Nivolumab in July 2017 based on data from the CHECKMATE 142 Trial (4). Stage IV Colorectal Cancer with $\mathrm{dMMR}$ can now be treated with immune checkpoint blockade (ICB) to induce durable tumor responses even after progression on standard chemotherapy agents with good tolerance of these drugs. However, only $\sim 5 \%$ of patients with mCRC are dMMR/MSI-H. What about the other $95 \%$ of patients with mCRC with proficient 
mismatch repair (pMMR) or microsatellite stability (MSS)?

This review will provide a summary on checkpoint immunotherapies in colorectal cancer with a focus on reporting advances and challenges in $\mathrm{pMMR} / \mathrm{MSS}$ tumors.

\section{ICB}

The two immune checkpoint pathways of highest clinical significant are PD-1/PDL-1 and CTLA4. CTLA4 engagement suppresses the tumor-specific immune response by inhibiting $\mathrm{T}$ cell proliferation and activation (5). Blockade of this pathway stimulates the adaptive anti-tumor immune response and ultimately tumor regression (6). Another mechanism that allows tumors to evade the immune system is tumor up-regulation of programmed death ligand-1 (PDL-1) which binds to coinhibitory programmed death receptor-1 (PD-1) on T cells. The PDL-1/PD-1 interaction negatively regulates T-cell receptor (TCR) signaling by decreasing $\mathrm{T}$ cell proliferation and cytokine production (7). Monoclonal antibodies directed against PD-1, PDL-1 and CTLA4 have been developed to overcome these tumor-mediated negative regulatory signals and thereby allow tumor-reactive $\mathrm{T}$ cells to mount an effective antitumor response.

\section{Success of ICB in mCRC: microsatellite instability selects for response}

Since the success of treatment with anti-CTLA4 antibodies and anti-PD-1 antibodies in refractory metastatic melanoma, there has been an explosion of clinical trials evaluating responses in nearly every other tumor type. One early example of efficacy in colon cancer was reported in the first in-human phase I trial of PD-1 antibody (MDX1106) in refractory solid tumors (8). In this initial dose escalation trial, 39 patients with advanced metastatic melanoma, CRC, castrate-resistant prostate cancer, nonsmall cell lung cancer (NSCLC) or renal cell carcinoma (RCC) received a single infusion of MDX-1106 with those having evidence of clinical benefit eligible for repeat therapy. Of the 14 patients with CRC, one achieved a CR persisting 3+ years; this tumor demonstrated $\mathrm{dMMR}$ with PD-L1 + lymphohistiocytic infiltrates surrounding tumor cells interposed with CD3+, PD-1 infiltrating T cells (9). In multiple subsequent studies, the only patients with mCRC who seemed to benefit from this class of drug were those with $\mathrm{dMMR}$, those with microsatellite stable (MSS) or proficient MMR (pMMR) had virtually no response with either PD-1 blockade alone or in combination with CTLA4 inhibitor (10-12). The most representative trial of this group was Keynote-016, a phase II study evaluating efficacy of Pembrolizumab in 41 patients with progressive metastatic carcinoma with or without $\mathrm{dMMR}$. Of the ten evaluable patients with dMMR CRC, 4 (40\%) had a partial response (PR) and an additional $5(50 \%)$ had stable disease (SD) at 12 weeks where none of the pMMR patients had an objective response, only 2 (11\%) with stable disease (12).

Microsatellite instability results from impaired DNA mismatch repair due to mutation or hypermethylation of mismatch repair genes ( $M L H 1, M S H 2, M S H 6$ or PMS2). This defect leads to accumulation of insertions and deletions in DNA repeat sequences (microsatellites) that ultimately result in increased number of frameshift or other somatic mutations (13). These dMMR tumors have multiple qualities that increase the likelihood that they would respond to ICBs that have been demonstrated in other tumor types which have robust responses to this treatment class, such as melanomas and NSCLC. These qualities which seem to be biomarkers of response, include high tumor mutational burden (TMB), PD1 expression and a high density of CD8+ tumor infiltrating lymphocytes (TILs).

Multiple studies in different tumor types have demonstrated that high TMB is a marker for response to ICB. The Cancer Genome Atlas Network published their genome wide analysis of 276 CRC samples, stratifying samples into two groups, those with mutation rates $<8.24 / 10^{6}$ (non-hypermutated) and those with mutation rates $>12 / 10^{6}$ (hypermutated). Seventy-seven percent of hypermutated tumors were MSI-H (23 of 30). The rate of frameshift mutations, which is thought to generate tumor neoantigens, was also 50-fold higher in hypermutated tumors than in non-hypermethylated tumors $(14,15)$. Genome-scale analysis of patient with dMMR colorectal cancers demonstrate that these tumors can harbor thousands of somatic mutations, more so than even NSCLC and melanoma which harbor an average 147 and 135 nonsynonymous mutations per tumor respectively (16). In Keynote-016, Le et al. reported a mean of 1,782 somatic mutations per tumor in $\mathrm{dMMR} \mathrm{c} / \mathrm{w} 73$ mutations per tumor in PMMR (12).

Deficient MMR colorectal tumors have also been demonstrated a higher density of CD8+ TILs (17-19). Multiple studies have demonstrated a positive prognostic value of TILs in colorectal cancer. There is also data correlating higher T-cell density (TCD) with response 


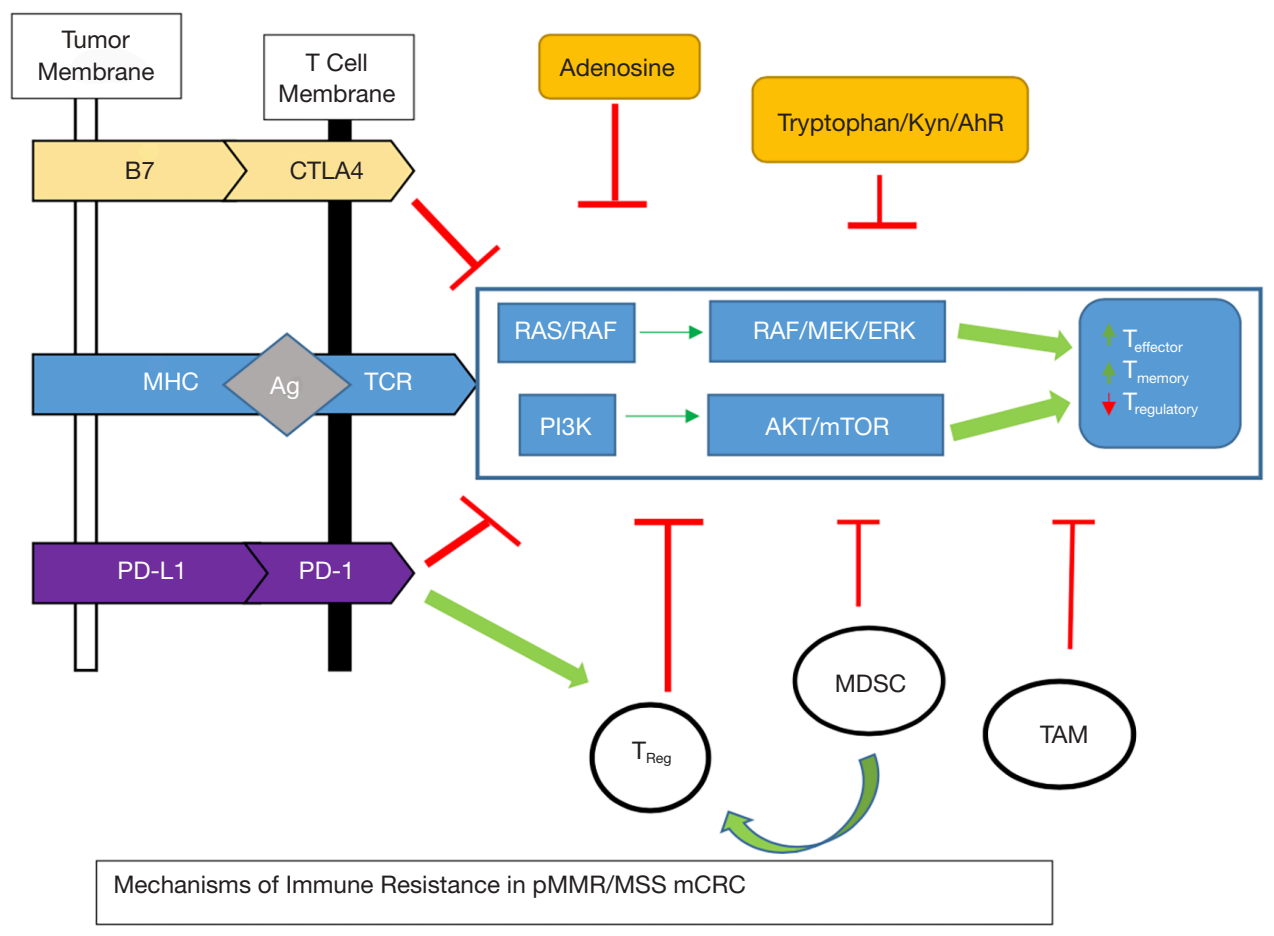

Figure 1 Mechanisms of immune resistance in pMMR/MSS mCRC. pMMR, proficient mismatch repair; MSS, microsatellite stable; CRC, colorectal cancer.

to immune checkpoint blockage. A retrospective review reported by Chakrabarti et al. of 12 patients with $\mathrm{dMMR}$ mCRC treated with Pembrolizumab noted that CD3+ and CD8+ TCD scores were higher in responders versus nonresponders and in those with longer duration of disease control. Moreover, in Keynote-016, tumors with dMMR contained a greater density of CD8-positive lymphoid cells, especially prominent at the invasive fronts of the tumors (12).

Mlecnik et al. reported the genomic differences in patients with dMMR vs. pMMR. Analysis of gene expression profiles of MSI-H and MSS samples demonstrated more heterogeneous pattern amongst the latter group and that the 951 differentially regulated genes were mainly associated with immune related functions. The profiles of MSI-H tumors included higher expression of T-helper 1 cells, cytotoxic genes, cytokines and chemokines. Interestingly, a small subgroup of hypermutated MSS patients was identified with this high intratumoral immune gene expression. These patients had improved survival patterns that were closer to their MSI-H counterparts. Beyond microsatellite instability, potentially actionable alterations were also enriched in MSI-H/hypermutated tumors compared with standard MSS tumors (86\% vs. $37 \%$,
$\mathrm{P}<0.001)$, including BRAF V600E (22\% vs. 5\%), BRCA1/ BRCA2 alterations (22\% vs. $1 \%$ ), and NTRK fusions ( $8 \%$ vs. $1 \%)$. PIK3CA and PTEN oncogenic mutations were both significantly enriched in MSI-H versus MSS mCRC (41\% vs. $16 \%, \mathrm{P}<0.01 ; 35 \%$ vs. $5 \%, \mathrm{P}<0.01)(20)$.

\section{Factors impeding success of ICB in pMRR/MSS CRC}

The attributes present in $\mathrm{d} M M \mathrm{R}$ colorectal cancers which induce tumor regression when exposed to ICB are unfortunately not inherently present in their pMMR counterparts. CRC with pMMR are characterized by low number of tumor mutations (low TMB), neoantigens and infiltrating immune effector cells with increased activity of immunosuppressive pathways. These factors all heighten tumor evasion from adaptive immunity and provide increased resistance mechanisms to immune checkpoint monotherapy (Figure 1). Strategies to overcome immune resistance by combining immune checkpoint therapy with additional agents aimed at increasing tumor antigen presentation, effector cell recognition of tumor antigens, generating a more immunogenic tumor microenvironment 
and decreasing immunosuppressive signaling are being evaluated in preclinical and clinical trials.

\section{Increasing neoantigens and antigen presentation}

One tool to increase exposure of tumor-associated antigens to the immune system with subsequent destruction of cancer cells is through cancer vaccines or bi-specific antibodies. Combining these agents with immune checkpoint inhibition would likely further augment the immune response. The ideal tumor antigens are those that have higher expression in tumor cells with relatively low or no expression on normal cells to minimize toxicity. Carcinoembryonic antigen (CEA) is one such antigen, with overexpression in $>80 \%$ of colorectal cancers with relatively little expression in normal tissues (21). Cibisatamab (CEA CD3 TCB,RG7802, RO6958688) is a novel T-cell bispecific antibody targeting CEA on tumor cells and CD3 on T cells. Early results from a phase $\mathrm{Ia} /$ Ib trial of cibisatamab as a single agent and in combination with atezolizumab (anti-PDL1) in CEA positive, treatment refractory tumors demonstrated a manageable safety profile (diarrhea, infusion reaction and fever) and some preliminary evidence of efficacy (22). Of the $31 \mathrm{mCRC}$ patients receiving monotherapy at CEA-TCB doses $>60 \mathrm{mg}$, 14 patients $(45 \%)$ had disease control, 2 with PR by RECIST v1.1. Among patient receiving dual therapy, 9 of $11(82 \%)$ mCRC patients treated at doses shown to induce tumor lesion inflammation by CT ( $80-160 \mathrm{mg}$ ) had disease control, 2 with PR. This study is estimated to be complete December 2019 with further results pending. Another bispecific antibody currently in development is MGD007 with dual affinity for anti-glycoprotein A33, which is expressed on colon cancer cells, and CD3. Preclinical studies demonstrated activity against colorectal cancer cells expressing gpA33 and it is currently being evaluated in phase I clinical trial in combination with a novel anti-PD1 antibody (23). A mRNA vaccine encoding four commonly found KRAS mutations in solids tumors including mCRC (mRNA-5671, Merck) has been developed and is currently in phase I trial in combination with pembrolizumab (24). Several other trials are underway evaluating the combination of immune check point blockade and cancer vaccines (24-28).

Oncolytic viruses activate the innate immune system by selectively infecting tumor cells and causing cell lysis with release of tumor specific antigens. Several viruses are under investigation both as single agents and in combination with ICB. Pexa-Vec is a thymidine kinase gene-inactivated oncolytic vaccinia virus engineered for the expression of transgenes encoding human granulocyte-macrophage colony-stimulating factor (GM-CSF) and $\beta$-galactosidase. In a phase I trial of a single IV infusion of Pexa-Vec in 23 patients with advanced solid tumors, including mCRC, dose dependent antitumor effect was noted without significant effect in normal tissues (29). Preliminary results from a phase I/II trial of Pexa-Vec in combination with durvalumab (anti-PD1) in patients with MSS mCRC and MSI-H mCRC refractory to prior PD-1 monotherapy were presented at GI ASCO 2019 (30). The combination showed a favorable safety profile; no dose limiting toxicities or grade 4-5 adverse events were observed. Further results regarding efficacy are pending.

The concept of an immunogenic cell death (ICD) has also been explored as a pathway to augment effect of ICB. This refers to a type of cell death characterized by translocation of calrecticulin (CRT), extracellular secretion of ATP and binding of high mobility group B1 (HMGB1) to toll-like receptor 4 (TLR4) which causes robust cytokine release and activation of antigen-presenting dendritic cells (DCs) and CD8+ TILs (31). Certain chemotherapy agents, radiation and ablation techniques have all demonstrated the capacity to induce ICD which has generated interest in combining these treatment modalities with PD-1 or CTLA4 inhibition. Pertinent to colorectal cancer, both 5 fluorouracil $(5 \mathrm{FU})$ and oxaliplatin have demonstrated the capacity to generate ICD. $5 \mathrm{FU}$ has been shown to be cytotoxic on myeloid-derived suppressor cells (MDSC), which are known to inhibit the function of CD8+ T cells, thereby promoting $\mathrm{T}$-cell dependent antitumor responses in vivo (32). Oxaliplatin stimulates pro-apoptotic CRT exposure and HMGB1 release in human colon cancer cell lines $(33,34)$. Oxaliplatin also causes upregulation of PD-L1 on DCs which further supports potential benefit of immune check point/chemotherapy combination (35).

Various studies investigating combination therapy with chemotherapy and ICBs. The addition of atezolizumab to chemotherapy + bevacizumab improved PFS and OS compared to chemotherapy + bevacizumab in metastatic NSCLA leading to FDA approval of this combination in first line therapy (36). Several trials are underway evaluating inhibitors of PD-1/PD-L1 and CTLA-4 pathways in combination with chemotherapy +/- vascular endothelial growth factor inhibitor (VEGFI) in pMMR mCRC. The phase I/II MEDITREME Trial, is evaluating the combination of FOLFOX combined with durvalumab plus 
tremelimumab as first line treatment in mCRC. Early results reported by Fumet et al. demonstrate acceptable safety in the 9 patients enrolled in the phase I component with disease control in 7 patients (4 PR, 3 SD) (37). The international, multicenter, open-label phase II/III CheckMate-9x8 will evaluate the safety and efficacy of Nivolumab in combination with standard-of-care (SOC) chemotherapy vs. SOC alone in first-line treatment for mCRC (38). SOC include standard 5FU backbone chemotherapy with oxaliplatin or irinotecan and biologic agent such as VEGFI. The BACCI Trial is a phase II randomized, double-blind placebo-controlled study of capecitabine/bevacizumab + atezolizumab vs. placebo in refractory $\mathrm{mCRC}$ that has completed accrual with preliminary results presented at the ESMO 2019 conference. The study reach its prespecified primary endpoint with significantly longer PFS in patients randomized to receive atezolizumab and is the first positive study co-targeting ICB and VEGF pathway. In MSS patients, HR for PFS was 0.67 (0.44-1.03), ORR $4.35 \%$ vs. $8.54 \%$ and 12 months OS was $43 \%$ vs. $52 \%$, HR $0.94, \mathrm{P}=0.4(39,40)$. The results of the phase II MODUL study, however, evaluating $5 \mathrm{FU} /$ bevacizumab with or without atezolizumab as maintenance following 16 weeks of induction treatment with FOLFOX demonstrated no improvement in PFS or OS with the addition of the antiPD-L1 inhibitor at 18 months of follow-up (41).

Radiation combined with ICB is another avenue by which investigators are seeking to overcome immune resistance pathways. Radiation induces ICD as demonstrated by release of ATP, HMGB-1 and CRT with increased production of IFN-1 (42). The well documented abscopal effect demonstrated in multiple tumor types whereby radiation to a representative tumor site triggers regression of metastatic tumor distant from the radiated site is driven by immune-mediated anti-tumor effect (43-45). Tumorinfiltrating/associated lymphocytes were increased post chemoradiotherapy in rectal cancer patients (46). A phase II study of pembrolizumab plus radiotherapy or ablation is being conducted in $\mathrm{pMMR} \mathrm{mCRC}$ patients after at least 2 treatment lines (47). In the radiation cohort, patients started pembrolizumab one week after the completion of palliative radiation to a metastatic site. The combination was relatively safe with no grade 3 or 4 drug-related AEs reported, 1 of 11 patients (9\%) achieved a PR, none in the ablation cohort. Additional patients were accrued to the radiation arm. Another phase IB study of pembrolizumab in combination with stereotactic body radiotherapy (SBRT) for restectable liver $\mathrm{mCRC}$ is underway with initial safety data presented at GI ASCO 2019 revealed no signal of increased immunotherapy-related toxicity; no grade 3 or 4 immunotherapy AEs reported (48). The combination of dual checkpoint blockade against both the CTLA4 and PD-1/PD-L1 pathway is likely to be even more synergistic with radiation based on pre-clinical models (49). A phase II study of durvalumab plus tremelimumab following palliative hypofractionated radiation in patients with MSS mCRC who have progressed on standard chemotherapy regimens is currently underway (50).

Epigenetic modulation by mechanisms such as histone modification and DNA methylation are mechanisms causing decreased tumor-associated antigens expression. Inhibiting these pathways with histone deacetylase (HDAC) or DNA methyltransferase (DNMT) inhibitors in combination with ICB is postulated to promote anti-tumor immunity. Significant inhibition of tumor growth and prolongation of survival was observed in CT26 colorectal cancer-bearing mouse model after treatment with combination of PD-1 blockade and low-dose decitabine (51). A phase II study of pembrolizumab in combination with azacitidine enrolled 31 patients with MSS chemotherapy refractory mCRC. Of 30 evaluable patients, only 1 achieved PR (ORR 3\%), 3 patients with SD and the study was stopped early for futility (52). Dual epigenetic therapy in combination with ICB in CT26 colorectal cancer-bearing mouse model compared with ICB alone dramatically improved tumor responses with $\mathrm{CR}$ in $>80 \%$ of tumor-bearing mice due to increased reduction of MDSCs (53). ENCORE 601, a phase $\mathrm{Ib} / \mathrm{II}$ dose escalation study of entinostat (HDAC inhibitor) with pembrolizumab in NSCLC with an expansion cohort in colorectal cancer is currently underway.

\section{Combining mechanisms of ICB}

Preclinical studies have demonstrated synergistic effects in anti-tumor immunity with combinations of PD-1/PD-L1 and CTLA 4 inhibition (54,55). This combination of ICB blockade has also translated into increased clinical efficacy as demonstrated in phase III clinical trials in other tumor types such as melanoma, as well as $\mathrm{dMMR}$ mCRC patients in the nivolumab plus ipilimumab cohort of CHeckMate-142, leading to FDA approval of dual therapy in this population $(56,57)$. By contrast, patients with $\mathrm{pMMR}$ treated with combination nivolumab/ipilimumab in CheckMate-142 had no significant response. Despite this, interest has continued in combination PD-1/PD-L1 and CTLA-4 inhibition using novel agents. CCTG CO.26 is a phase II randomized 
trial evaluating the combination of durvalumab with tremelimumab $v s$. best supportive care (BSC) in patients with $\mathrm{mCRC}$ who had failed all standard regimens (58). The study randomized 179 patients and with a median follow-up of 15.2 months, though there was no difference in median PFS (1.8 vs. 1.9 months) there was a statistically significant survival benefit of 6.6 vs. 4.1 months favoring durvalumab/ tremelimumab, HR 1.01 (90\% CI, 0.76-1.34). Grade 3/4 abdominal pain, fatigue, lymphocytosis and eosinophilia were significantly higher in the experimental arm but there was significantly less deterioration on EROTC QLQ-C30 physical function in treated patients. Though this is a positive trial statistically, it must be noted that the alpha of 0.1 used in this study allows for a $10 \%$ risk of false-positive results. These outcomes should be verified with additional studies, ideally a randomized, placebo controlled trial.

Novel ICI Monalizumab (IPH2201) is a humanized antibody targeting NKG2a receptors expressed on tumor infiltrating cytotoxic NK and CD8+ T-cells which bind HLA-E on tumor cells, blunting immune responses (59). Segal et al. reported results of the first in-human dose escalation of monalizumab with durvalumab with an expansion cohort in patients with mCRC (60). Of the 40 patients with MSS-CRC treated in the expansion cohort, 37 were evaluable with $3 \mathrm{PR}$ and $11 \mathrm{SD}$, including 3 patients with tumor reduction who continued therapy for $>200$ days. DCR was $24 \%$ at 16 weeks. The combination was well tolerated, no DTLs reported and MTD was not reached in escalation and represents a promising potential therapy in mCRC warranting further study.

In addition to PD-1 and CTLA-4, additional coinhibitory receptors have been identified as potential targets for drug development, namely Lymphocyte-activation gene 3 (LAG3), T cell immunoglobulin-3 (TIM-3), and T cell immunoglobulin and ITIM domain (TIGIT) (61).

Lymphocyte activation gene 3 (LAG3) is an additional immune checkpoint pathway that negatively regulates effector $\mathrm{T}$ cell function, is a marker for $\mathrm{T}$ cell exhaustion (decreased cytokine production and $\mathrm{T}$ cell proliferation) and has an essential role in preventing autoimmunity. It is expressed on activated human T cells, NK cells and also expressed on tumor infiltrating Tregs further suggesting a role in suppressing antitumoral immunity as well (62). LAG3 blockade has even been demonstrated to induce superior $\mathrm{T}$ cell activation compared with PD1/PD-L1 inhibition in cell culture (63). The strong synergy between LAG3 and PDL1 inhibitory pathways in promoting tolerance to both self and tumor antigens has been demonstrated in mouse models suggesting a superior immune response with dual blockade of these pathways (64). To that end, several drugs modulating the LAG3-MHC II interaction have been developed and are currently under clinical evaluation in combination with anti-PD1 agents in solid tumors, including metastatic pMMR colorectal cancer. Results from a phase I/IIa clinical trial of anti-LAG3 monoclonal antibody Relatlimab (BMS-986016) + nivolumab in previously treated metastatic melanoma with progression on prior anti-PD(L)1 therapy demonstrated similar safety profile to nivolumab monotherapy with disease control rate of $45 \%$, ORR $16 \%$ (65). Based on these promising results, a phase II/III trial is underway with Nivolumab +/- Relatlimab first-line in metastatic melanoma (66). There is a phase II trial underway evaluating Nivolumab + Relatlimab in patients with MSS mCRC (67).

TIGIT and TIM-3 are additional inhibitory receptors expressed on NK cells, CD8+ T-cells and Tregs which participate in antitumoral immunity. Pharmacologic agents have been developed targeting these pathways. AB154 is a fully humanized antibody against TIGIT with preclinical data demonstrating synergistic effects with antiPD1 antibodies increasing $\mathrm{T}$ cell activation and IFN $\gamma$ secretion (68) and is currently in phase I trial in advanced solid malignancies including MSS CRC in combination with PD-1 blockade (69). TSR-022 is an anti-TIM-2 Monoclonal Ab currently being tested in phase I trial for solid tumors (AMBER trial) with an mCRC expansion cohort in combination with an anti-PD-1 antibody (70).

\section{Downregulation of immunosuppressive signaling patbways: targeting MAPK, WNT}

The mitogen-activated protein kinase pathway (MAPK) is involved in proliferation, apoptosis and cell motility. Increased MAPK signalizing has demonstrated a role in tumor immune evasion by impairing recruitment and function of TILs. Inhibition of this pathway downregulates immunosuppressive cytokines as well as PD-L1 and VEGFA while promoting apoptosis markers (71). Preclinical studies demonstrated that inhibition of MAPK signaling pathway could decrease immune resistance by upregulating HLA molecule expression, potentiate anti-tumor T-cells, increase TILs and work synergistically in combination with antiPD-L1 (71,72). In a phase IB clinical trial, atezolizumab was combined with dose escalating doses of cobimetinib in 23 patients with MSS CRC and demonstrated ORR of $17 \%$ (4PR, 5SD) with good tolerance (73). Despite these early 
results, a subsequent phase III open-label, randomized trial of atezolizumab plus or minus cobimetinib versus regorafenib in patients with chemotherapy-refractory mCRC (IMblaze370), which enrolled 363 patients, did not demonstrate any differences in PFS, OS or ORR between the three cohorts. Less than $3 \%$ of patients in any group achieving an objective response and a significant increase in grade $3+$ adverse events in the combination group $v s$. atezolizumab alone (74). Selumetinib (AZD6244) is another MEK-inhibitor under evaluation in combination with durvalumab and tremelimumab in solid tumors with a colon cancer cohort for safety and efficacy in the SELECT-4 trial (75).

The Wnt/ $\beta$-catenin signaling pathway is frequently dysregulated in colorectal cancers and is highlighted by Grasso et al. as a key mechanism of immune evasion in colorectal cancer (76). Molecular profiling of 1,211 primary CRC tumors through pooled databases demonstrated that both MSS and MSI-high cancer have lower T cell infiltration when molecular events that up-regulate the WNT pathway are present. Galinpepimut-S is a multivalent WT1 peptide vaccine that is being tested in combination with pembrolizumab in patients with advanced cancers, including mCRC a phase I/II, open-label, multicenter, multiarm study (77).

\section{Modulating tumor microenvironment}

The vascular endothelial growth factor pathway functions as an important negative regulator of the tumor microenvironment by inhibiting $T$ cell function, stimulating proliferation of Tregs and inhibiting development of DCs (78-80). Preclinical data in a mouse model demonstrated increased anti-tumor effect in vivo from dual with PD-1 and VEGFR2 blockade with increased tumor growth inhibition $v s$. blockade of either individual pathway (81). In addition to monoclonal antibodies directed against VEGFR such as bevacizumab, small molecule TKIs with anti-VEGFR activity such as cabozantinib, and regorafenib, which have also demonstrated preclinical immune anti-tumoral activity in combination with PD-1/PD-L1, are also being evaluated in early phase trials. The results of one such phase Ib trial, REGONIVO, evaluating regorafenib plus nivolumab in patients with metastatic refractory gastric or CRC, was presented at ASCO 2019. Total of 50 patients were enrolled, 25 with $\mathrm{mCRC}$ who had received median of 3 prior treatments, and treated with Regorafenib 80-160 mg daily days 1-21 of a 28-day cycle and Nivolumab $3 \mathrm{mg} / \mathrm{kg}$ days 1 and 15. In the dose expansion cohort of Regorafenib
$120 \mathrm{mg}$, dose was decreased to $80 \mathrm{mg}$ due to frequent grade III skin toxicities. Objective tumor response was observed in 19 (38\%) patients including $11 \mathrm{MSS}$ gastric cancers and 7 MSS CRC; response rate of 29\% in MSS CRC. Grade 3 or higher treatment related adverse events occurred in $34 \%$ of patients though felt to be manageable at reduced regorafenib dose of $80 \mathrm{mg}$ daily. Correlative data analysed from pre- and post-treatment tumor samples demonstrated a reduction of Treg fraction with tumor response (82).

Interventions that would decrease activity of negative immunomodulatory cells such as Tregs, MDSCs and tumorassociated macrophages (TAM) would further support an immunogenic tumor microenvironment. Novel targets include TGF $\beta$, CSF1 pathway, CC Chemokine 5 (CCR5), and IL-5. NIS793 is an anti-TGF $\beta$ antibody currently undergoing phase I evaluation with PD-1 blockade (83). Preclinical studies of anti-CSF1R antibody demonstrates activity against TAMs and have mostly been investigated in the treatment of tenosynovial giant tumors but have also been combined with nivolumab in phase I trial of mCRC and pancreatic cancer (84-86). A phase I trial is underway combining CSF-1R tyrosine kinase inhibitor, pexidartinib, with durvalumab in mCRC (87).

\section{Suppressive metabolic tumor environment}

A suppressive metabolic tumor environment contributes to ineffective T-cell function and therefore is a manner by while tumor cells can escape immune recognition. A metabolically permissive TME fosters T-cell effector function, proliferation, cytokine and mitochondrial function. A metabolically restrictive TME suppresses T cell effector function and proliferation, cytokine production and promotes immunosuppressive $\mathrm{T}$ regulatory cells (Tregs). Adenosine signaling through the hypoxia-CD39-CD73A2aR pathway has emerged as key regulator of tumor immunity (88). Extracellular adenosine generated by CD39 and CD73 dampens the immune response by suppressing effector cell function and promotes immunosuppressive regulatory cells (89). The $\mathrm{A} 2 \mathrm{a}$ receptor (A2aR) is a highaffinity receptor expressed on T cells, natural killer (NK) cells, macrophages, monocytes, and DCs; its activation inhibits effector cell activation, cytokine production and seems to trigger increased expression of immune checkpoint pathways such as PD-1, CTLA-4 and LAG-3 $(88,89)$. Drugs targeting multiple steps in this pathway have been developed, against CD39, CD73 and the A2aR, and are currently being evaluated in phase I/II clinical trials as 
single agents and in combination with anti-PD-1 drugsOleclumab (anti-CD73), CPI-444, PBF-509, MK-3814 and AZD4635 target A2aR. Early results from one such trial was presented at ASCO 2019-in the first in-human phase I trial in colorectal and pancreatic cancer patients, oleclumab alone and in combination with durvalumab demonstrated acceptable toxicity and some clinical activity with $\mathrm{PR}$ and SD observed in 1 and 2 of 21 CRC patients respectively (57).

Another important pathway involved in maintaining a suppressive TME is the Tryptophan-kynurenine-aryl hydrocarbon receptor (Trp-Kyn-AhR) pathway which is a mediator of immunosuppression in T-cell inflamed tumors. One target in this pathway was indoleamine 2,3 dioxygenase 1 (IDO1), a principle enzyme of Trp catabolism. IDO1 is expressed by stromal cells of the TME and is induced by IFN $\gamma$ due to CD8+ T-cell infiltration as part the immunosuppression mechanism. Epacadostat, an inhibitor of IDO1, demonstrated promising results in multiple early phase clinical trials in refractory solid tumors when combined with PD-1 checkpoint inhibitors (90). However, in a phase III trial in melanoma, ECHO-301/Keynote-252, the combination of epacadostat with pembrolizumab failed to improve PFS and ORR vs. Pembrolizumab alone (91). Epacadostat is the only drug in this pathway that is undergoing clinical trial evaluation in colon cancers. There are currently several Phase I/II trials underway in pMSS colorectal cancer with Epacadostat in combination with PD-1 inhibitors +/- other agents [ECHO-206 Epacadostat, Azacitidine and Pembrolizumab in advanced solid tumors, ECHO-207/KEYNOTE-723 Epacadostat,
Pembrolizumab and mFOLFOX6 63 (91-93)].

\section{Conclusions}

In striking contrast to their hypermutated counterparts with $\mathrm{d} M M R$, metastatic colorectal cancers with proficient DNAmismatch repair are largely unresponsive to monotherapy with ICB. These tumors are characterized by immune resistance patterns such as low level of somatic mutations that serve as neoantigens, a poorly permissive tumor microenvironment with increased immunosuppressive cells and upregulation of immunosuppressive cytokines as well as decreased tumor infiltrating cells. Mlecnik et al. identified a subgroup of MSS tumors with genomic profiles that reflected higher expression of T-helper1 cells, immunostimulatory cytokines and chemokines such as IFN- $\gamma$, IL-15, CXCL16. Patients with higher "immunoscore" reflecting higher densities of CD8+ and $\mathrm{CD} 3+\mathrm{T}$ cells in the tumor center and invasive margin, regardless of MMR status, had improved survival (20). Strategies to produce these results among otherwise inherently low immunoscore MSS patients is the focus of intense research. Novel targets are currently being evaluated in combination with single or dual ICB in an effort to modify immune resistance and overcome tumor evasion without excess off-target immune stimulation triggering autoimmune toxicities (Table 1). Ultimately, the goal is to increase tumor specific neoantigens that will lead to recruitment and proliferation of CD3+/CD8+ TILs while blunting the immunoinhibitory response. Taking advantage of the ICD

Table 1 Selected ongoing trials of ICB combination in MSS/pMMR CRC

\begin{tabular}{|c|c|c|c|c|c|}
\hline Study name/ID & Intervention & Target & Phase & CRC study population & Status \\
\hline \multicolumn{6}{|c|}{ Bi-specific antibodies/cancer vaccines/adoptive T cell therapy } \\
\hline NCT03866239 & $\begin{array}{l}\text { Cibisatamab + Atezolizumab; after } \\
\text { pretreatment w/obinutuzumab }\end{array}$ & CEA-CD3; PDL-1 & $\mathrm{lb}$ & $\begin{array}{l}\text { MSS mCRC, w/ high } \\
\text { CEACAM5 expression }\end{array}$ & Recruiting \\
\hline NCT02650713 & Cibisatamab +/- Atezolizumab & CEA-CD3; PDL-1 & $\mathrm{la} / \mathrm{lb}$ & MSS mCRC & $\begin{array}{l}\text { Active not } \\
\text { recruiting }\end{array}$ \\
\hline NCT03531632 & MGD007 + MGA012 & gpA33-CD3; PD-1 & $\mathrm{I} / \mathrm{II}$ & $\begin{array}{l}\text { Relapsed /refractory } \\
\mathrm{mCRC}\end{array}$ & $\begin{array}{l}\text { Active, not } \\
\text { recruiting }\end{array}$ \\
\hline NCT03948763 & mRNA-5671 +/- Pembrolizumab & $\begin{array}{l}\text { mRNA-567/V941; vaccine; } \\
\text { PD-1 }\end{array}$ & I & MSS mCRC & Recruiting \\
\hline NCT03953235 & $\begin{array}{l}\text { Personalized Neoantigen Cancer } \\
\text { Vaccine + Nivolumab + Ipilimumab }\end{array}$ & $\begin{array}{l}\text { GRT-C903 (prime vaccine); } \\
\text { GRT-R904 (boost vaccine); } \\
\text { PD-1; CTLA4 }\end{array}$ & I & MSS mCRC & Recruiting \\
\hline
\end{tabular}

Table 1 (continued) 
Table 1 (continued)

\begin{tabular}{|c|c|c|c|c|c|}
\hline Study name/ID & Intervention & Target & Phase & CRC study population & Status \\
\hline $\begin{array}{l}\text { NCT03206073 } \\
\text { (NCl-17-C-0092) }\end{array}$ & $\begin{array}{l}\text { Pexa-Vec }(J X-594)+\text { Durvalumab + } \\
\text { tremelimumab }\end{array}$ & $\begin{array}{l}\text { Oncolytic viral vaccine; PD1; } \\
\text { CTLA4 }\end{array}$ & $\mathrm{I} / \mathrm{II}$ & $\begin{array}{l}\text { MSI-H mCRC (prev } \\
\text { PD1/PDL1) or MSS } \\
\text { mCRC }\end{array}$ & Recruiting \\
\hline $\begin{array}{l}\text { NCT03050814 } \\
\text { (QUILT-2.004) }\end{array}$ & $\begin{array}{l}\text { FOLFOX/bevacizumab +/- Ad-CEA } \\
+ \text { Avelumab }\end{array}$ & $\begin{array}{l}\text { A Chemotherapy; Adenoviral } \\
\text { vector; PDL-1 }\end{array}$ & II & MSS mCRC & Recruiting \\
\hline NCT02636036 (SPICE) & Enadenotucirev + Nivolumab & $\begin{array}{l}\text { Tumor selective chimeric } \\
\text { adenovirus; PD-1 }\end{array}$ & I & MSS mCRC & Recruiting \\
\hline NCT02757391 & $\begin{array}{l}\text { Autologous CD8+ T Cells + } \\
\text { Pembrolizumab }\end{array}$ & Adoptive T cells; PD-1 & I & $\mathrm{mCRC}$ & $\begin{array}{l}\text { Active, not } \\
\text { recruiting }\end{array}$ \\
\hline \multicolumn{6}{|l|}{ Chemotherapy } \\
\hline $\begin{array}{l}\text { NCT03202758 } \\
\text { (MEDITREME) }\end{array}$ & $\begin{array}{l}\text { Durvalumab + Tremelimumab + } \\
\text { FOLFOX }\end{array}$ & PD-1; CTLA4; chemotherapy & $\mathrm{I} / \mathrm{II}$ & MSS mCRC & Recruiting \\
\hline NCT02873195 (BACCI) & $\begin{array}{l}\text { Capecitabine + Bevacizumab +/- } \\
\text { Atezolizumab }\end{array}$ & Chemotherapy; VEGF; PDL-1 & II & MSS mCRC & $\begin{array}{l}\text { Active, not } \\
\text { recruiting }\end{array}$ \\
\hline $\begin{array}{l}\text { NCT02375672(HCRN } \\
\text { Gl14-186) }\end{array}$ & Pembrolizumab; FOLFOX & PD-1; chemotherapy & II & $\mathrm{mCRC}$ & $\begin{array}{l}\text { Active, not } \\
\text { recruiting }\end{array}$ \\
\hline $\begin{array}{l}\text { NCT03414983 } \\
\text { (Checkmate 9x8) }\end{array}$ & $\begin{array}{l}\text { FOLFOX + Bevacizumab +/- } \\
\text { Nivolumab }\end{array}$ & Chemotherapy; VEGFR; PD-1 & $\| / I I I$ & $\mathrm{mCRC}$ & $\begin{array}{l}\text { Active, not } \\
\text { recruiting }\end{array}$ \\
\hline \multicolumn{6}{|l|}{ VEGF/multikinase inhibitors } \\
\hline NCT03946917 & Toripalimab (JS001) + Regorafenib & PD-1; VEGF/multikinase & $\mathrm{I} / \mathrm{II}$ & MSS mCRC & Recruiting \\
\hline NCT03977090 & $\begin{array}{l}\text { Genolimzumab (GB226) + } \\
\text { Fruquintinib }\end{array}$ & PD-1; VEGF & $\mathrm{lb}$ & $\mathrm{mCRC}$ & Recruiting \\
\hline \multicolumn{6}{|l|}{ Radiation } \\
\hline NCT03927898 & Toripalimab + SBRT & PD-1; SBRT & II & MSS mCRC & Recruiting \\
\hline NCT03122509 & $\begin{array}{l}\text { Durvalumab + Tremelimumab + } \\
\text { Radiation or Ablation }\end{array}$ & $\begin{array}{l}\text { PD-1; CTLA4; radiation or } \\
\text { ablation }\end{array}$ & II & MSS mCRC & Recruiting \\
\hline NCT02888743 & $\begin{array}{l}\text { Durvalumab + Tremelimumab +/-; } \\
\text { high or low dose RT }\end{array}$ & $\begin{array}{l}\text { PD-1; CTLA4; } \\
\text { high or low dose RT }\end{array}$ & II & MSS mCRC & $\begin{array}{l}\text { Active not } \\
\text { recruiting }\end{array}$ \\
\hline NCT03007407 & $\begin{array}{l}\text { Durvalumab + Tremelimumab after } \\
\text { Radiation }\end{array}$ & PD-1; CTLA4 & II & MSS mCRC & $\begin{array}{l}\text { Active not } \\
\text { recruiting }\end{array}$ \\
\hline $\begin{array}{l}\text { NCT04030260 } \\
\text { (REGONIVO) }\end{array}$ & $\begin{array}{l}\text { Regorafenib + Nivolumab + } \\
\text { Radiotherapy }\end{array}$ & VEGF; PD-1; radiation & II & MSS mCRC & Recruiting \\
\hline \multicolumn{6}{|l|}{ Epigenetic modulation } \\
\hline $\begin{array}{l}\text { NCT03993626 } \\
\text { (CAROSELL) }\end{array}$ & CXD101 + Nivolumab & HDAC; PD-1 & $\mathrm{I} / \mathrm{II}$ & MSS mCRC & $\begin{array}{l}\text { Active, not } \\
\text { recruiting }\end{array}$ \\
\hline $\begin{array}{l}\text { NCT02959437 } \\
(\mathrm{ECHO}-206)\end{array}$ & $\begin{array}{l}\text { Azacitidine + Pembrolizumab + } \\
\text { Epacadostat }\end{array}$ & $\begin{array}{l}\text { DNA Methyltransferase; } \\
\text { PD-1; IDO-1 }\end{array}$ & $\mathrm{I} / \mathrm{II}$ & MSS mCRC & $\begin{array}{l}\text { Active, not } \\
\text { recruiting }\end{array}$ \\
\hline $\begin{array}{l}\text { NCT02437136 } \\
\text { (ENCORE 601) }\end{array}$ & Entinostat + Pembrolizumab & HDAC; PD-1 & $\mathrm{lb} / \mathrm{II}$ & MSS mCRC & $\begin{array}{l}\text { Active, not } \\
\text { recruiting }\end{array}$ \\
\hline
\end{tabular}

Table 1 (continued) 
Table 1 (continued)

\begin{tabular}{|c|c|c|c|c|c|}
\hline Study name/ID & Intervention & Target & Phase & CRC study population & Status \\
\hline \multicolumn{6}{|c|}{ Combination immune checkpoint blockade } \\
\hline \multicolumn{6}{|c|}{ PD-1/PDL-1 Inhibitors + CTLA4 } \\
\hline NCT03982121 & $\begin{array}{l}\text { Intratumoral Ipilimumab + GLA-SE } \\
\text { and Nivolumab Chemotherapy }\end{array}$ & $\begin{array}{l}\text { CTLA 4; toll-like receptor } 4 \\
\text { (TLR4); PD-1; FOLFOX }\end{array}$ & I & $\mathrm{mCRC}$ & Recruiting \\
\hline \multicolumn{6}{|c|}{ PD-1/PDL-1 + Natural Killer Cell Targets } \\
\hline NCT02671435 & $\begin{array}{l}\text { Monalizumab + Cetuximab +/- } \\
\text { Durvalumab }\end{array}$ & NKG2a; EGFR; PDL-1 & $\mathrm{I} / \mathrm{II}$ & MSS MCRC RAS WT & Recruiting \\
\hline \multicolumn{6}{|c|}{ PD-1/PDL-1 + Co-inhibitor Receptor Blockade (LAG3/TIM-3/TIGIT) } \\
\hline NCT03628677 & $\mathrm{AB} 154+\mathrm{AB} 122$ & TIGIT; PD-1 & I & $\mathrm{mCRC}$ & Recruiting \\
\hline NCT03642067 & Relatlimab + Nivolumab & LAG3; PD-1 & II & MSS mCRC & Recruiting \\
\hline NCT02817633 & TSR-022 + TSR-042 & TIM-3; PD-1 & I & $\mathrm{mCRC}$ & Recruiting \\
\hline \multicolumn{6}{|l|}{ Targeting MAPK/WNT/PI3K } \\
\hline NCT02586987 & $\begin{array}{l}\text { Selumetinib + Durvalumab + } \\
\text { Tremelimumab }\end{array}$ & MEK; PDL-1; CTLA4 & I & MSS mCRC & $\begin{array}{l}\text { Active, not } \\
\text { recruiting }\end{array}$ \\
\hline $\begin{array}{l}\text { NCT03374254 } \\
\text { (KEYNOTE-651) }\end{array}$ & $\begin{array}{l}\text { Pembrolizumab + Binimetinib or } \\
\text { Pembrolizumab + Chemo +/- } \\
\text { Binimetinib }\end{array}$ & $\begin{array}{l}\text { PD-1; MEK; } \\
\text { FOLFOX/FOLFIRI }\end{array}$ & I & MSS mCRC & Recruiting \\
\hline NCT03271047 & $\begin{array}{l}\text { Binimetinib + Nivolumab +/- } \\
\text { Ipilimumab }\end{array}$ & MEK; PD-1; CTLA4 & $\mathrm{I} / \mathrm{II}$ & $\begin{array}{l}\text { MSS mCRC with RAS } \\
\text { mutation }\end{array}$ & $\begin{array}{l}\text { Active, not } \\
\text { recruiting }\end{array}$ \\
\hline $\begin{array}{l}\text { NCT03377361 } \\
\text { (Checkmate 9N9) }\end{array}$ & $\begin{array}{l}\text { Nivolumab + Trametinib +/- } \\
\text { Ipilimumab }\end{array}$ & PD-1; MEK; CTLA4 & $\mathrm{I} / \mathrm{II}$ & $\begin{array}{l}\text { MSS and MSI-H } \\
\text { mCRC }\end{array}$ & $\begin{array}{l}\text { Active, not } \\
\text { recruiting }\end{array}$ \\
\hline NCT03711058 & Copanlisib + Nivolumab & PI3K; PD-1 & $\mathrm{I} / \mathrm{II}$ & MSS mCRC & Recruiting \\
\hline NCT03761914 & Galinpepimut-S + Pembrolizumab & WT1 Protein; PD-1 & $\mathrm{I} / \mathrm{II}$ & $\mathrm{mCRC}$ & Recruiting \\
\hline \multicolumn{6}{|c|}{ Targeting negative immunomodulatory cells (Tregs, MDSC, TAM) } \\
\hline NCT03631407 & $\begin{array}{l}\text { Vicriviroc (MK-7690) + } \\
\text { Pembrolizumab }\end{array}$ & Chemokine Receptor 5 PD-1 & II & MSS mCRC & $\begin{array}{l}\text { Active, not } \\
\text { recruiting }\end{array}$ \\
\hline NCT02947165 & NIS793 + PDR001 & TGF-b; PD-1 & $\mathrm{I} / \mathrm{II}$ & MSS mCRC & Recruiting \\
\hline $\begin{array}{l}\text { NCT02777710 } \\
\text { (MEDIPLEX) }\end{array}$ & Durvalumab + Pexidartinib & PDL-1; CSF-1R TKI & I & $\mathrm{mCRC}$ & $\begin{array}{l}\text { Active, not } \\
\text { recruiting }\end{array}$ \\
\hline \multicolumn{6}{|c|}{ Modulating metabolic tumor environment (arginase, adenosine, tryptophan pathways) } \\
\hline NCT03549000 & NZV930 +/- PDR001+/- NIR178 & CD73; PD-1; adenosine A2AR & $\mathrm{I} / \mathrm{II}$ & MSS mCRC & Recruiting \\
\hline $\begin{array}{l}\text { NCT03085914 (ECHO- } \\
\text { 207/KEYNOTE-723) }\end{array}$ & $\begin{array}{l}\text { Epacadostat + Pembrolizumab + } \\
\text { Chemotherapy }\end{array}$ & IDO-1; PD-1; FOLFOX & $\mathrm{I} / \mathrm{II}$ & $\begin{array}{l}\text { Solid metastatic } \\
\text { tumors (phase I) }\end{array}$ & $\begin{array}{l}\text { Active, not } \\
\text { recruiting }\end{array}$ \\
\hline NCT02903914 & INCB001158 +/- Pembrolizumab & $\begin{array}{l}\text { Arginase Inhibitor } \\
\text { PD-1 }\end{array}$ & $\mathrm{I} / \mathrm{II}$ & MSS and MSI mCRC & Recruiting \\
\hline \multicolumn{6}{|l|}{ Additional mechanisms } \\
\hline NCT02834052 & Poly-ICLC + Pembrolizumab & TLR3 Agonist; PD-1 & $\mathrm{I} / \mathrm{II}$ & MSS mCRC & Recruiting \\
\hline NCT03647839 & Nivolumab + BBI608 or BNC105 & $\begin{array}{l}\text { PD-1; Tubulin Polymerization } \\
\text { Inhibitor (vascular disruption) } \\
\text { STAT3 Inhibitor }\end{array}$ & II & MSS mCRC & Recruiting \\
\hline
\end{tabular}

pMMR, proficient mismatch repair; MSS, microsatellite stable; CRC, colorectal cancer; ICB, immune checkpoint blockade. 
induced by radiation injury, certain chemotherapy, biologic agents such as VEGFI are strategies to increase tumor antigen presentation. The majority of these trials remain in phase I/II level but there has been some positive preliminary data that will guide future phase III trial development. The early results of the BACCI Trial demonstrated a statistically significant PFS benefit when capecitabine/bevacizumab was combined with atezolizumab vs. capecitabine/bevacizumab alone (40). Although the benefit was modest in this phase II trial, correlative analysis might help identify predictors of benefit. The REGONIVO Trial recent presented at ASCO in June 2019 demonstrated a 29\% response rate in patients with MSS mCRC treated with Regorafenib and Nivolumab who had received a median of 3 lines of prior therapy. Thirty four percent of patients experienced grade 3 or higher toxicities though they were the toxicities that are well known in clinical practice with regorafenib alone as an approved 3rd line agent in refractory mCRC without significant additional toxicity due to nivolumab. The CCTG CO.26 is another recently published phase II trial reporting preliminary positive results with a statistically significant survival benefit of 2.5 months favoring durvalumab/ tremelimumab over BSC in treatment refractory mCRC though alpha of 0.1 increases risk for false-positive results (58). The phase III IMBlaze 370 trial evaluating MEK1/2 inhibitor cobimetinib + atezolizumab against, standard of care in 3rd line mCRC, single - agent regorafenib was surprisingly negative despite promising results reported in the phase I trial which reported ORR of $17 \%$ and median OS of an impressive 10 months $(73,74)$. There was no PFS or OS benefit over regorafenib alone, with a trend toward superiority favoring this standard of care control. While the results of the IMBlaze 370 trial might seem like a cautionary tale against moving toward phase III trials based on these somewhat modest phase I results, the reality is that there is a great deal of heterogeneity in this population and these larger studies are needed with correlative testing to identify the subgroup of $\mathrm{pMMR}$ that will ultimately benefit from these therapies and guide future trials.

\section{Acknowledgments}

Funding: None.

\section{Footnote}

Provenance and Peer Review: This article was commissioned by the Guest Editor (Khaldoun Almhanna) for the series
"GI malignancies and Immunotherapy" published in Translational Gastroenterology and Hepatology. The article was sent for external peer review organized by the Guest Editor and the editorial office.

Conflicts of Interest: The author has completed the ICMJE uniform disclosure form (available at http://dx.doi. org/10.21037/tgh.2020.03.08). The series "GI malignancies and Immunotherapy" was commissioned by the editorial office without any funding or sponsorship. The author has no other conflicts of interest to declare.

Ethical Statement: The author is accountable for all aspects of the work in ensuring that questions related to the accuracy or integrity of any part of the work are appropriately investigated and resolved.

Open Access Statement: This is an Open Access article distributed in accordance with the Creative Commons Attribution-NonCommercial-NoDerivs 4.0 International License (CC BY-NC-ND 4.0), which permits the noncommercial replication and distribution of the article with the strict proviso that no changes or edits are made and the original work is properly cited (including links to both the formal publication through the relevant DOI and the license). See: https://creativecommons.org/licenses/by-nc-nd/4.0/.

\section{References}

1. Siegel RL, Miller KD, Jemal A. Cancer statistics, 2019. CA Cancer J Clin 2019;69:7-34.

2. Heidelberger C, Chaudhuri NK, Danneberg P, et al. Fluorinated pyrimidines, a new class of tumour-inhibitory compounds. Nature 1957;179:663-6.

3. Hodi FS, O'Day SJ, McDermott DF, et al. Improved Survival with Ipilimumab in Patients with Metastatic Melanoma. N Engl J Med 2010;363:711-23.

4. Overman MJ, McDermott R, Leach JL, et al. Nivolumab in patients with metastatic DNA mismatch repair-deficient or microsatellite instability-high colorectal cancer (CheckMate 142): an open-label, multicentre, phase 2 study. Lancet Oncol 2017;18:1182-91.

5. Alegre ML, Frauwirth KA, Thompson CB. T-cell regulation by CD28 and CTLA-4. Nat Rev Immunol 2001;1:220-8.

6. Fong L, Small EJ. Anti-Cytotoxic T-Lymphocyte Antigen-4 Antibody: The First in an Emerging Class of Immunomodulatory Antibodies for Cancer Treatment. J 
Clin Oncol 2008;26:5275-83.

7. Blank C, Gajewski TF, Mackensen A. Interaction of PDL1 on tumor cells with PD-1 on tumor-specific T cells as a mechanism of immune evasion: implications for tumor immunotherapy. Cancer Immunol Immunother 2005;54:307-14.

8. Brahmer JR, Drake CG, Wollner I, et al. Phase I Study of Single-Agent Anti-Programmed Death-1 (MDX-1106) in Refractory Solid Tumors: Safety, Clinical Activity, Pharmacodynamics, and Immunologic Correlates. J Clin Oncol 2010;28:3167-75.

9. Lipson EJ, Sharfman WH, Drake CG, et al. Durable Cancer Regression Off-Treatment and Effective Reinduction Therapy with an Anti-PD-1 Antibody. Clin Cancer Res 2013;19:462-8.

10. Topalian SL, Hodi FS, Brahmer JR, et al. Safety, Activity, and Immune Correlates of Anti-PD-1 Antibody in Cancer. N Engl J Med 2012;366:2443-54.

11. Overman MJ, Kopetz S, McDermott RS, et al. Nivolumab \pm ipilimumab in treatment (tx) of patients (pts) with metastatic colorectal cancer (mCRC) with and without high microsatellite instability (MSI-H): CheckMate-142 interim results. J Clin Oncol 2016;34:3501.

12. Le DT, Uram JN, Wang H, et al. PD-1 Blockade in Tumors with Mismatch-Repair Deficiency. N Engl J Med 2015;372:2509-20.

13. Boland CR, Goel A. Microsatellite instability in colorectal cancer. Gastroenterology 2010;138:2073-87.e3.

14. Cancer Genome Atlas Network. Comprehensive molecular characterization of human colon and rectal cancer. Nature 2012;487:330-7.

15. Maby P, Galon J, Latouche JB. Frameshift mutations, neoantigens and tumor-specific CD8(+) $\mathrm{T}$ cells in microsatellite unstable colorectal cancers. Oncoimmunology 2015;5:e1115943.

16. Vogelstein B, Papadopoulos N, Velculescu VE, et al. Cancer Genome Landscapes. Science 2013;339:1546-58.

17. Smyrk TC, Watson P, Kaul K, et al. Tumor-infiltrating lymphocytes are a marker for microsatellite instability in colorectal carcinoma. Cancer 2001;91:2417-22.

18. Michael-Robinson JM, Biemer-Hüttmann A, Purdie $\mathrm{DM}$, et al. Tumour infiltrating lymphocytes and apoptosis are independent features in colorectal cancer stratified according to microsatellite instability status. Gut 2001;48:360-6.

19. Dolcetti R, Viel A, Doglioni C, et al. High Prevalence of Activated Intraepithelial Cytotoxic T Lymphocytes and Increased Neoplastic Cell Apoptosis in Colorectal
Carcinomas with Microsatellite Instability. Am J Pathol 1999;154:1805-13.

20. Mlecnik B, Bindea G, Angell HK, et al. Integrative Analyses of Colorectal Cancer Show Immunoscore Is a Stronger Predictor of Patient Survival Than Microsatellite Instability. Immunity 2016;44:698-711.

21. Tiernan JP, Perry SL, Verghese ET, et al. Carcinoembryonic antigen is the preferred biomarker for in vivo colorectal cancer targeting. Br J Cancer 2013;108:662-7.

22. Tabernero J, Melero I, Ros W, et al. Phase Ia and Ib studies of the novel carcinoembryonic antigen (CEA) T-cell bispecific (CEA CD3 TCB) antibody as a single agent and in combination with atezolizumab: Preliminary efficacy and safety in patients with metastatic colorectal cancer (mCRC). J Clin Oncol 2017;35:3002.

23. Moore PA, Shah K, Yang Y, et al. Development of MGD007, a gpA33 x CD3-Bispecific DART Protein for T-Cell Immunotherapy of Metastatic Colorectal Cancer. Mol Cancer Ther 2018;17:1761-72.

24. A Study of mRNA-5671/V941 as Monotherapy and in Combination With Pembrolizumab (V941-001). Available online: https://clinicaltrials.gov/ct2/show/NCT03948763

25. A Study of a Personalized Neoantigen Cancer Vaccine. Available online: https://clinicaltrials.gov/ct2/show/ NCT03794128

26. MGD007 Combined With MGA012 in Relapsed/ Refractory Metastatic Colorectal Cancer. Available online: https://clinicaltrials.gov/ct2/show/NCT03531632

27. QUILT-3.050: NANT Colorectal Cancer (CRC) Vaccine: Combination Immunotherapy in Subjects With Recurrent or Metastatic CRC. Available online: https://clinicaltrials. gov/ct2/show/NCT03169777

28. Romero P, Banchereau J, Bhardwaj N, et al. The Human Vaccines Project: A roadmap for cancer vaccine development. Sci Transl Med 2016;8:334ps9.

29. Breitbach CJ, Burke J, Jonker D, et al. Intravenous delivery of a multi-mechanistic cancer-targeted oncolytic poxvirus in humans. Nature 2011;477:99-102.

30. Morelli MP, Xie C, Brar G, et al. A phase I/II study of pexa-vec oncolytic virus in combination with immune checkpoint inhibition in refractory colorectal cancer: Safety report 2019;37:646-.

31. Zhou J, Wang G, Chen Y, et al. Immunogenic cell death in cancer therapy: Present and emerging inducers. J Cell Mol Med 2019;23:4854-65.

32. Vincent J, Mignot G, Chalmin F, et al. 5-Fluorouracil selectively kills tumor-associated myeloid-derived 
suppressor cells resulting in enhanced T cell-dependent antitumor immunity. Cancer Res 2010;70:3052-61.

33. Panaretakis T, Kepp O, Brockmeier U, et al. Mechanisms of pre-apoptotic calreticulin exposure in immunogenic cell death. EMBO J 2009;28:578-90.

34. Tesniere A, Schlemmer F, Boige V, et al. Immunogenic death of colon cancer cells treated with oxaliplatin. Oncogene 2010;29:482-91.

35. Tel J, Hato SV, Torensma R, et al. The chemotherapeutic drug oxaliplatin differentially affects blood DC function dependent on environmental cues. Cancer Immunol Immunother 2012;61:1101-11.

36. Socinski MA, Jotte RM, Cappuzzo F, et al. Atezolizumab for First-Line Treatment of Metastatic Nonsquamous NSCLC. N Engl J Med 2018;378:2288-301.

37. Fumet JD, Isambert N, Hervieu A, et al. 85PEvaluation of the safety and the tolerability of durvalumab plus tremelimumab combined with FOLFOX in metastatic colorectal cancer (MEDITREME). Ann Oncol 2018;29:x24-x38.

38. Tabernero J, Yoshino T, Cohn AL, et al. Open-label phase II/III study of nivolumab plus standard of care versus standard of care for first-line treatment of metastatic colorectal cancer: Checkmate-9X8. J Clin Oncol 2019;37:TPS718-TPS.

39. Mettu NB, Twohy E, Ou F, et al. BACCI: A phase II randomized, double-blind, multicenter, placebocontrolled study of capecitabine (C) bevacizumab (B) plus atezolizumab (A) or placebo $(\mathrm{P})$ in refractory metastatic colorectal cancer (mCRC): An ACCRU network study. Ann Oncol 2019;30:v198-v252.

40. Mettu NB, Niedzwiecki D, Boland PM, et al. BACCI: A phase II randomized, double-blind, placebo-controlled study of capecitabine bevacizumab plus atezolizumab versus capecitabine bevacizumab plus placebo in patients with refractory metastatic colorectal cancer. J Clin Oncol 2018;36:TPS873.

41. Grothey A, Tabernero J, Arnold D, et al. Fluoropyrimidine $(\mathrm{FP})+$ bevacizumab $(\mathrm{BEV})$ + atezolizumab vs $\mathrm{FP} / \mathrm{BEV}$ in BRAFwt metastatic colorectal cancer (mCRC): Findings from Cohort 2 of MODUL - a multicentre, randomized trial of biomarker-driven maintenance treatment following first-line induction therapy. Ann Oncol 2018;29.

42. Golden EB, Frances D, Pellicciotta I, et al. Radiation fosters dose-dependent and chemotherapyinduced immunogenic cell death. Oncoimmunology 2014;3:e28518.

43. Postow MA, Callahan MK, Barker CA, et al. Immunologic
Correlates of the Abscopal Effect in a Patient with Melanoma. N Engl J Med 2012;366:925-31.

44. Demaria $\mathrm{S}, \mathrm{Ng} \mathrm{B}$, Devitt $\mathrm{ML}$, et al. Ionizing radiation inhibition of distant untreated tumors (abscopal effect) is immune mediated. Int J Radiat Oncol Biol Phys 2004;58:862-70.

45. Antoniades J, Brady LW, Lightfoot DA. Lymphangiographic demonstration of the abscopal effect in patients with malignant lymphomas. Int J Radiat Oncol Biol Phys 1977;2:141-7.

46. Lim SH, Chua W, Cheng C, et al. Effect of neoadjuvant chemoradiation on tumor-infiltrating/associated lymphocytes in locally advanced rectal cancers. Anticancer Res 2014;34:6505-13.

47. Segal NH, Kemeny NE, Cercek A, et al. Non-randomized phase II study to assess the efficacy of pembrolizumab (Pem) plus radiotherapy (RT) or ablation in mismatch repair proficient (pMMR) metastatic colorectal cancer (mCRC) patients. J Clin Oncol 2016;34:3539.

48. Turk AA, Lubner SJ, Uboha NV, et al. A phase Ib study of pembrolizumab (Pem) in combination with stereotactic body radiotherapy (SBRT) for resectable liver metastatic colorectal cancer (CRC). J Clin Oncol 2019;37:680.

49. Twyman-Saint Victor C, Rech AJ, Maity A, et al. Radiation and dual checkpoint blockade activate non-redundant immune mechanisms in cancer. Nature 2015;520:373-7.

50. Study of Durvalumab and Tremelimumab After Radiation for Microsatellite Stable Metastatic Colorectal Cancer Progressing on Chemotherapy. Available online: https:// clinicaltrials.gov/ct2/show/NCT03007407

51. Yu G, Wu Y, Wang W, et al. Low-dose decitabine enhances the effect of PD-1 blockade in colorectal cancer with microsatellite stability by re-modulating the tumor microenvironment. Cell Mol Immunol 2019;16:401-9.

52. Lee JJ, Sun W, Bahary N, et al. Phase 2 study of pembrolizumab in combination with azacitidine in subjects with metastatic colorectal cancer. J Clin Oncol 2017;35:3054.

53. Kim K, Skora AD, Li Z, et al. Eradication of metastatic mouse cancers resistant to immune checkpoint blockade by suppression of myeloid-derived cells. Proc Natl Acad Sci U S A 2014;111:11774-9.

54. Spranger S, Koblish HK, Horton B, et al. Mechanism of tumor rejection with doublets of CTLA-4, PD-1/PD-L1, or IDO blockade involves restored IL-2 production and proliferation of CD8(+) $\mathrm{T}$ cells directly within the tumor microenvironment. J Immunother Cancer 2014;2:3.

55. Curran MA, Montalvo W, Yagita H, et al. PD-1 and 
CTLA-4 combination blockade expands infiltrating T cells and reduces regulatory $\mathrm{T}$ and myeloid cells within B16 melanoma tumors. Proc Natl Acad Sci U S A 2010;107:4275-80.

56. Wolchok JD, Chiarion-Sileni V, Gonzalez R, et al. Overall Survival with Combined Nivolumab and Ipilimumab in Advanced Melanoma. N Engl J Med 2017;377:1345-56.

57. Overman MJ, LoRusso P, Strickler JH, et al. Safety, efficacy and pharmacodynamics (PD) of MEDI9447 (oleclumab) alone or in combination with durvalumab in advanced colorectal cancer (CRC) or pancreatic cancer (panc). J Clin Oncol 2018;36:4123.

58. Chen EX, Jonker DJ, Kennecke HF, et al. CCTG CO.26 trial: A phase II randomized study of durvalumab (D) plus tremelimumab (T) and best supportive care (BSC) versus $\mathrm{BSC}$ alone in patients (pts) with advanced refractory colorectal carcinoma (rCRC). J Clin Oncol 2019;37:481.

59. André P, Denis C, Soulas C, et al. André P, Denis C, Soulas C, et al. Anti-NKG2A mAb Is a Checkpoint Inhibitor that Promotes Anti-tumor Immunity by Unleashing Both T and NK Cells. Cell 2018;175:1731-43.e13.

60. Segal NH, Naidoo J, Curigliano G, et al. First-in-human dose escalation of monalizumab plus durvalumab, with expansion in patients with metastatic microsatellite-stable colorectal cancer. J Clin Oncol 2018;36:3540.

61. Anderson AC, Joller N, Kuchroo VK. Lag-3, Tim-3, and TIGIT: Co-inhibitory Receptors with Specialized Functions in Immune Regulation. Immunity 2016;44:989-1004.

62. Andrews LP, Marciscano AE, Drake CG, et al. LAG3 (CD223) as a cancer immunotherapy target. Immunol Rev 2017;276:80-96.

63. Lichtenegger FS, Rothe M, Schnorfeil FM, et al. Targeting LAG-3 and PD-1 to Enhance T Cell Activation by Antigen-Presenting Cells. Front Immunol 2018;9:385.

64. Woo SR, Turnis ME, Goldberg MV, et al. Immune inhibitory molecules LAG-3 and PD-1 synergistically regulate $\mathrm{T}$-cell function to promote tumoral immune escape. Cancer Res 2012;72:917-27.

65. Ascierto PA, Melero I, Bhatia S, et al. Initial efficacy of anti-lymphocyte activation gene-3 (anti-LAG-3; BMS986016 ) in combination with nivolumab (nivo) in pts with melanoma (MEL) previously treated with anti-PD-1/PDL1 therapy. J Clin Oncol 2017;35:9520.

66. Lipson EJ, Long GV, Tawbi H, et al. CA224-047: A randomized, double-blind, phase II/III study of relatlimab (anti-LAG-3) in combination with nivolumab (anti-PD-1) versus nivolumab alone in previously untreated metastatic or unresectable melanoma. Ann Oncol 2018;29:viii442- viii66.

67. Study of Nivolumab and Relatlimab in Patients With Microsatellite Stable (MSS) Advanced Colorectal Cancer. Available online: https://clinicaltrials.gov/ct2/show/ NCT03642067

68. Anderson AE, Becker A, Yin F, et al. Abstract A124: Preclinical characterization of AB154, a fully humanized anti-TIGIT antibody, for use in combination therapies. Cancer Immunol Res 2019;7:A124-A.

69. A Study to Evaluate the Safety and Tolerability of AB154 in Participants With Advanced Malignancies. Available online: https://clinicaltrials.gov/ct2/show/NCT03628677

70. A Phase 1 Study of TSR-022, an Anti-TIM-3 Monoclonal Antibody, in Patients With Advanced Solid Tumors (AMBER). Available online: https://clinicaltrials.gov/ct2/ show/NCT02817633

71. Liu L, Mayes PA, Eastman S, et al. The BRAF and MEK Inhibitors Dabrafenib and Trametinib: Effects on Immune Function and in Combination with Immunomodulatory Antibodies Targeting PD-1, PD-L1, and CTLA-4. Clin Cancer Res 2015;21:1639-51.

72. Ebert PJR, Cheung J, Yang Y, et al. MAP Kinase Inhibition Promotes T Cell and Anti-tumor Activity in Combination with PD-L1 Checkpoint Blockade. Immunity 2016;44:609-21.

73. Bendell JC, Kim TW, Goh BC, et al. Clinical activity and safety of cobimetinib (cobi) and atezolizumab in colorectal cancer (CRC). J Clin Oncol 2016;34:3502.

74. Eng C, Kim TW, Bendell J, et al. Atezolizumab with or without cobimetinib versus regorafenib in previously treated metastatic colorectal cancer (IMblaze370): a multicentre, open-label, phase 3 , randomised, controlled trial. Lancet Oncol 2019;20:849-61.

75. A Study to Assess the Safety, Tolerability and Antitumour Activity of Ascending Doses of Selumetinib in Combination With MEDI4736 and Selumetinib in Combination With MEDI4736 and Tremelimumab in Patients With Advanced Solid Tumours. Available online: https://clinicaltrials.gov/ct2/show/NCT02586987

76. Grasso CS, Giannakis M, Wells DK, et al. Genetic Mechanisms of Immune Evasion in Colorectal Cancer. Cancer Discov 2018;8:730-49.

77. Galinpepimut-S in Combination With Pembrolizumab in Patients With Selected Advanced Cancers. Available online: https://clinicaltrials.gov/ct2/show/NCT03761914

78. Terme M, Pernot S, Marcheteau E, et al. VEGFA-VEGFR Pathway Blockade Inhibits Tumor-Induced Regulatory T-cell Proliferation in Colorectal Cancer. Cancer Res 
2013;73:539-49.

79. Motz GT, Santoro SP, Wang LP, et al. Tumor endothelium FasL establishes a selective immune barrier promoting tolerance in tumors. Nat Med 2014;20:607-15.

80. Gabrilovich D, Ishida T, Oyama T, et al. Vascular Endothelial Growth Factor Inhibits the Development of Dendritic Cells and Dramatically Affects the Differentiation of Multiple Hematopoietic Lineages In Vivo. Presented in part at the Keystone Symposium "Cellular and Molecular Biology of Dendritic Cells," Santa Fe, NM, March 3-9, 1998, and at the annual meeting of the American Association for Cancer Research, March 28-April 1, 1998. Blood 1998;92:4150-66.

81. Yasuda S, Sho M, Yamato I, et al. Simultaneous blockade of programmed death 1 and vascular endothelial growth factor receptor 2 (VEGFR2) induces synergistic antitumour effectin vivo. Clin Exp Immunol 2013;172:500-6.

82. Fukuoka S, Hara H, Takahashi N, et al. Regorafenib plus nivolumab in patients with advanced gastric (GC) or colorectal cancer (CRC): An open-label, dose-finding, and dose-expansion phase 1b trial (REGONIVO, EPOC1603). J Clin Oncol 2019;37:2522.

83. Mariathasan S, Turley SJ, Nickles D, et al. TGF $\beta$ attenuates tumour response to PD-L1 blockade by contributing to exclusion of $\mathrm{T}$ cells. Nature 2018;554:544-8.

84. Sankhala KK, Blay JY, Ganjoo KN, et al. A phase I/II dose escalation and expansion study of cabiralizumab (cabira; FPA-008), an anti-CSF1R antibody, in tenosynovial giant cell tumor (TGCT, diffuse pigmented villonodular synovitis D-PVNS). J Clin Oncol 2017;35:11078.

85. Wainberg ZA, Piha-Paul SA, Luke J, et al. First-inHuman Phase 1 Dose Escalation and Expansion of a Novel Combination, Anti-CSF-1 Receptor (cabiralizumab) Plus
Anti-PD-1 (nivolumab), in Patients With Advanced Solid Tumors. 2018. doi: 10.13140/RG.2.2.28962.53443.

86. Zhu Y, Knolhoff BL, Meyer MA, et al. CSF1/CSF1R blockade reprograms tumor-infiltrating macrophages and improves response to T-cell checkpoint immunotherapy in pancreatic cancer models. Cancer Res 2014;74:5057-69.

87. Evaluation of Safety and Activity of an Anti-PDL1 Antibody (DURVALUMAB) Combined With CSF-1R TKI (PEXIDARTINIB) in Patients With Metastatic/ Advanced Pancreatic or Colorectal Cancers. Available online: https://clinicaltrials.gov/ct2/show/NCT02777710

88. Leone RD, Emens LA. Targeting adenosine for cancer immunotherapy. J Immunother Cancer 2018;6:57.

89. Allard B, Longhi MS, Robson SC, et al. The ectonucleotidases CD39 and CD73: Novel checkpoint inhibitor targets. Immunol Rev 2017;276:121-44.

90. Labadie BW, Bao R, Luke JJ. Reimagining IDO Pathway Inhibition in Cancer Immunotherapy via Downstream Focus on the Tryptophan-Kynurenine-Aryl Hydrocarbon Axis. Clin Cancer Res 2019;25:1462-71.

91. Long GV, Dummer R, Hamid O, et al. Epacadostat plus pembrolizumab versus placebo plus pembrolizumab in patients with unresectable or metastatic melanoma (ECHO-301/KEYNOTE-252): a phase 3, randomised, double-blind study. Lancet Oncol 2019;20:1083-97.

92. Azacitidine Combined With Pembrolizumab and Epacadostat in Subjects With Advanced Solid Tumors (ECHO-206). Available online: https://ClinicalTrials.gov/ show/NCT02959437

93. A Study of Epacadostat in Combination With Pembrolizumab and Chemotherapy in Subjects With Advanced or Metastatic Solid Tumors (ECHO-207/ KEYNOTE-723). Available online: https://ClinicalTrials. gov/show/NCT03085914 doi: $10.21037 / \operatorname{tgh} .2020 .03 .08$

Cite this article as: Breakstone R. Colon cancer and immunotherapy—can we go beyond microsatellite instability? Transl Gastroenterol Hepatol 2021;6:12. 